\title{
Contrast Enhancement of Cross-Polarization OCT Images of Breast Cancer by Optical Coefficient Calculation
}

\author{
DOI: $10.17691 / \mathrm{stm} 2019.11,3.03$
}

Received May 16, 2019

E.V. Gubarkova, PhD, Senior Researcher, Scientific Laboratory of Optical Coherence Tomography, Institute of Experimental Oncology and Biomedical Technologies";

N.P. Paylova, Assistant, Scientific Laboratory of Optical Coherence Tomography, Institute of Experimental Oncology and Biomedical Technologies';

E.B. Kiseleva, PhD, Senior Researcher, Scientific Laboratory of Optical Coherence Tomography, Institute of Experimental Oncology and Biomedical Technologies';

D.A. Vorontsov, Oncology Surgeon, Oncology Department2;

A.A. Moiseev, PhD, Senior Researcher, Laboratory of Highly Sensitive Optical Measurements ${ }^{3}$;

A.A. Plekhanov, PhD Student, Institute of Experimental Oncology and Biomedical Technologies';

S.S. Kuznetsov, MD, DSc, Head of the Pathological Anatomy Department';

M.A. Sirotkina, PhD, Head of Scientific Laboratory of High-Resolution Microscopy and Gene Technologies, Institute of Experimental Oncology and Biomedical Technologies';

A.Y. Vorontsov, MD, PhD, Oncology Surgeon, Head of the Oncology Department';

N.D. Gladkova, MD, DSc, Professor, Head of the Scientific Laboratory of Optical Coherence Tomography, Institute of Experimental Oncology and Biomedical Technologies ${ }^{1}$

${ }^{1}$ Privolzhsky Research Medical University, 10/1 Minin and Pozharsky Square, Nizhny Novgorod, 603005, Russia;

${ }^{2}$ Nizhny Novgorod Regional Oncologic Dispensary, 11/1 Delovaya St., Nizhny Novgorod, 603126, Russia;

${ }^{3}$ Federal Research Center Institute of Applied Physics, Russian Academy of Sciences, 46 Ulyanova St.

Nizhny Novgorod, 603950, Russia

The aim of the study was to develop the techniques for contrast enhancement of cross-polarization OCT (CP OCT) images of breast cancer $(\mathrm{BC})$ by calculating optical attenuation coefficients of OCT signal to differentiate cancer subtypes and analyze polarization properties of tumor stroma.

Materials and Methods. The study involved ex vivo breast tissue samples after radical mastectomy. Scattering and polarization properties were studied using a high-speed spectral OCT unit suitable for cross-scattering recording with higher specificity enabling to differentiate breast connective tissue in health and cancer. We presented the findings of qualitative and quantitative analysis of en face CP OCT images of non-tumor breast cancer and two the most common BC types, which differ fundamentally by tumor stroma condition infiltrating ductal carcinoma of solid and sclerosing structures. Optical coefficients were calculated for contrast enhancement of CP OCT images: attenuation coefficient in a co-channel (coefficient 1) and a difference attenuation coefficient in co- and cross-channels (coefficient 2), which enabled both: to differ tumor tissue from non-tumor tissue, and differentiate two breast cancer types under study.

Results. En face CP OCT images of breast tissue without tumor are characterized by heterogeneity due to different scattering capacity of fat, glandular and connective tissue with predominantly low values of both optical coefficients. Infiltrating ductal BC of a solid structure is characterized by homogenous distribution of lowest values of both optical coefficients. In case of infiltrating ductal BC of a sclerosing structure, CP OCT images have the most heterogeneous and contrast OCT signal related to the tumor stroma dominated over parenchyma, and various degenerative changes are in stroma (fibrosis or hyalinosis).

Conclusion. The use of optical attenuation coefficients of OCT signal in two channels and en face color-coded mapping is an obvious example presenting CP OCT images of BC and an objective technique for OCT signal quantitative assessment. Moreover, imaging contrast enhances, it eases the differentiation of morphological BC subtypes and can be used in clinical settings.

Key words: cross-polarization optical coherence tomography; CP OCT; breast cancer; BC; attenuation coefficient; difference attenuation coefficient in co- and cross-channels; differential diagnosis; morphological breast cancer subtypes.

\section{Introduction}

Breast cancer $(\mathrm{BC})$ is one of the most common carcinomas (over a million of new cases are recorded every year) and the third leading cause of death among women (over 500 thousand deaths are registered every year) worldwide $[1,2]$. In Russia, BC ranks first in morbidity rate $(21.1 \%)$ and mortality rate $(16.4 \%)$ among malignancies in women [3]. BC is a genetically heterogeneous disease with various biomolecular

Corresponding author: Ekaterina V. Gubarkova, e-mail: kgybarkova@mail.ru 
and morphological characteristics [4], which enable to distinguish a number of disease subtypes; their early detection is essential when choosing the management and therapy for BC patients. Infiltrating ductal carcinoma (IDC) is the most common BC subtype (up to $75 \%$ of all cases) [5].

Morphological/molecular BC subtype is established in histological/immunohistological and genetic study of biopsy and operative specimens and has no alternative intra-operative assessment method. The development and application of in vivo high-resolution imaging techniques compatible with a morphological investigation can improve the quality of early and intra-operative BC diagnostics.

Rapidly developing diagnostic techniques and advances in BC therapy promote that sparing operations are increasingly frequently given preference in surgery. Therefore, there is a growing clinical demand for intra-operative reveal of "clean" surgical margin in lumpectomy (local tumor incision with a small healthy surrounding tissue area). The remains of the tumor sites increase the probability of local recurrence development that will require reoperation or radiotherapy $[6,7]$.

Current BC imaging techniques such as ultrasound, mammography, positron emission tomography, and magnetic resonance tomography can detect a tumor node size, and provide general morphological information [8-10]. However, the low spatial resolution fails to detect lesion less than $1 \mathrm{~mm}$ in size and record in vivo negative surgical margin. In addition, some morphological BC subtypes (such as carcinoma in situ and tumors with fibrous stroma) are difficult to image [11]. Currently, there are several available methods used to assess a surgical margin, however, all of them have some limitations. For example, widely used conventional microscopic methods such as express biopsy and cytologic screening of imprint smears have their errors and difficulties due to insufficient sampling, technical problems occurring when working with adipose tissue and additional time needed for investigation [12].

Optical coherence tomography (OCT) is a threedimensional promising technique of optical imaging, its resolution being up to micrometer units, to differentiate healthy tissue, benign and malignant tumor tissue including $B C[13,14]$, as well as to determine a precise intra-operative "clean" surgical margin in real-time mode [15-17]. For automated differentiation of healthy breast tissue from tumor tissue using conventional (non-polarization-sensitive) OCT we used and calculated optical refraction, dispersion, and attenuation coefficients [17, 18]. Heterogeneous distribution of high and low values of backscattering coefficient was found to be the characteristic for adipose tissue, which consists of "empty" lipid-filled cells and nuclei on the periphery. Mean values of a scattering coefficient and low values of an attenuation coefficient are typical for connective tissue. A high scattering coefficient with a high attenuation is characteristic for tumor tissue, since tumor cells are densely spaced making tissue be poorly permeable to light $[17,19]$. Nevertheless, less dense invasive tumors can be difficult to distinguish from healthy connective tissue due to similar optical refraction and scattering intensity indices [20, 21].

To enhance contrast imaging that can promote differential diagnostics of healthy and tumor breast tissue, to be used in clinical settings, we used OCT systems, which enable to obtain polarization-dependent [22, 23] and elastographic [24-26] information estimating, correspondingly, optical double refraction and elasticity of biological tissues. Using the systems, normal breast stroma was found to have significantly higher double refraction index compared to tumor stroma due to characteristic structure of the last one (the presence of degenerative changes of collagen fibers, their changed spatial orientation) [22]. Optical coherence elastography was demonstrated to have high application potential to detect the contrast between malignant and healthy breast tissues based on tissue compression deformability analysis [25, 26].

Collagen fibers comprise the base of healthy breast tissue stroma. Fiber condition (physical parameters) changes in BC. Different BC subtypes being characterized by specific structure of connective tissue and its certain ratio with tumor cells. Currently, all clinically used in vivo imaging techniques cannot give high-resolution evidence of breast connective tissue. The common structural OCT images of infiltrating BC subtypes of a solid structure are difficult to differentiate from infiltrating masses with fibrous or hyalinized stroma due to a similar high backscattering coefficient. Detection of cross-polarization properties of collagen fibers using real-time cross-polarization OCT (CP OCT) in solid and sclerosing $\mathrm{BC}$ subtypes will enable both to detect tumors and determine its subtype. Moreover, the criteria for quantitative OCT signal assessment to both differentiate $\mathrm{BC}$ subtypes and to real-time intra-operatively determine "clean" surgical margin have not been established yet.

The aim of the present study was to develop the techniques to enhance the contrast of CP OCT image of BC by calculating optical attenuation coefficients of the signal to differentiate cancer subtypes and analyze tumor stroma polarization properties.

\section{Materials and Methods}

Ex vivo breast tissue samples $(n=75)$ obtained in radical mastectomy in 52 patients aged 38-77 years were investigated by CP OCT. The study was approved by the Ethics Committee of Privolzhsky Research Medical University. The samples were delivered in phosphate buffer-wet gauze, at $7^{\circ} \mathrm{C}$, and were studied within 1-2 $\mathrm{h}$ after sampling.

Cross-polarization OCT system. A high-speed spectral 3D OCT system (Federal Research Center Institute of Applied Physics, Russian Academy of Sciences, Nizhny Novgorod) was applied in the study. 
The OCT setup characterised by central wavelength $1310 \mathrm{~nm}$ and spectral width of $100 \mathrm{~nm}$. Radiation power is $2 \mathrm{~mW}$, resulting in axial resolution of $15 \mu \mathrm{m}$, lateral resolution is $20 \mu \mathrm{m}$, scanning depth in the air is $1.7 \mathrm{~mm}$. Scanning speed is $20 \mathrm{kHz} / \mathrm{s}, 3 D$-image $2.4 \times 2.4 \times 1.25 \mathrm{~mm}$ in size was recorded within $26 \mathrm{~s}$. CP OCT setup was equipped with an end optical fiber probe, its outer objective diameter being $10 \mathrm{~mm}$, which was led to the studied tissue.

The OCT records simultaneously two conjugated images: in co- and cross-polarization channels [27]. The cross-polarization channel provides evident advantages for connective tissue study (in particular, collagen fibers, since some studies [28, 29] show that they have polarization properties).

The present study demonstrates CP OCT images as en face 2D images in co- and cross-polarization channels.

Morphological analysis. All tissue samples were histologically studied after CP OCT-scanning. Serial histological sections were made in the same orientation with CP OCT images. Serial sections both hematoxylin and eosin (H\&E) and Van-Gieson's were stained to determine breast cancer subtype and collagen fibers content, respectively.

All tissue samples were divided by a pathologist into 3 groups according to the morphological diagnosis and tumor structure characteristics [30]: non-tumor breast tissue $(n=14)$; infiltrative nonspecific (ductal) carcinoma: solid $(n=17)$ and sclerosing structures $(n=44)$. Totally, there were obtained and studied 150 pairs of CP OCT and histological images.

Quantitative analysis of en face CP OCT images. Quantitative analysis of scattering and polarization properties of tissue samples was performed by 3D OCT data. Each 3D OCT image contains 256×256 A-scans and presents OCT signal depth attenuation profile in each polarization. Attenuation coefficient of light was calculated by A-scans in co- and cross-channels.

For quantitative analysis of scattering tissue properties, an attenuation coefficient (coefficient 1) was used. It indicates photon attenuation rate in a cochannel, which can be caused by various reasons, such as absorption, side scattering or cross-polarization scattering. The method of the coefficient calculation reported in the paper [31].

To describe polarization properties a difference attenuation coefficient (coefficient 2) was used. It demonstrates the difference in photon scattering and absorption in tissues in co- and cross-polarization channels.

The consideration of the above-mentioned processes, the change of scanning beam intensity with depth $z$ can be presented as follows

$$
\begin{gathered}
I_{c o} \sim \exp (-2 z u), \\
I_{c r o s s} \sim \exp \left(-2 z\left(u-u_{D}\right),\right.
\end{gathered}
$$

where $I_{c o}, I_{\text {cross }}$ - a signal in co- and cross-channels, respectively; $u, u-u_{D}$ are the attenuation coefficients in corresponding channels.

Figures 1 (e), 2 (e), 3(e) show the examples of random A-scans from 3D data set in co- (blue color) and cross-channels (orange color) and demonstrate significant difference in OCT signal attenuation rate. A black dotted rectangle on A-scans indicates depth range $(80-720 \mu \mathrm{m})$, where optical coefficients were calculated.

2D (en face) color-coded maps were made based on obtained two optical coefficients (Figure 1-3 (f) and $(g)$ ). The range of values was adjusted so that colors most accurately indicate different tissue types (tumor and nontumor, fat and collagen).

To inter compare the maps in the study groups, it was suggested conventionally mark the areas with high values of coefficient 1 - over $1.5 \mathrm{~mm}^{-1}$, and coefficient 2 - over $0.5 \mathrm{~mm}^{-1}$ (green and yellow-red colors) and the areas with their low values: less than $1.5 \mathrm{~mm}^{-1}$ and less than $0.5 \mathrm{~mm}^{-1}$, respectively (light blue and blue colors). Constructed this way color-coded maps perfectly contrast cellular (by low values of the coefficients) and stromal (by high values of the coefficients) breast tissue components including those in different infiltrative $B C$ subtypes.

Optical coefficients were calculated for $150 \mathrm{CP}$ OCT images (two areas of interest of each sample), which included non-tumor breast tissue $(n=28)$, infiltrative ductal BC of solid $(n=34)$, and sclerosing $(n=88)$ structure.

\section{Results and Discussion}

\section{Visual analysis of scattering and polarization properties of breast tissue and morphological $B C$ subtypes by en face CP OCT images}

Non-tumor breast tissue. In norm, the mammary gland is formed of a terminal duct lobular unit, adipose and connective tissue (Figure 1 (a), (b)) that makes it heterogeneous exhibiting different scattering and polarization properties. It explains heterogeneous OCT signal distribution on structural en face CP OCT images of non-tumor breast tissue in co- and cross-polarization channels (Figure 1 (c), (d)). Breast adipose tissue is visualized as the accumulation of roundish structures with a low signal from cytoplasm, and a high signal from cell membrane. Connective tissue gives a high signal both in co- and cross-polarization channels due to arranged, parallel collagen fibers; however, a signal quickly attenuates in depth. A terminal duct lobular unit or a single dilated glandular duct is visualized as the lowsignal area in co- and cross-polarization channels.

Infiltrative ductal BC of solid structure. CP OCT technique enables to differentiate the subtypes of high grade infiltrative ductal BC characterized by a poor 


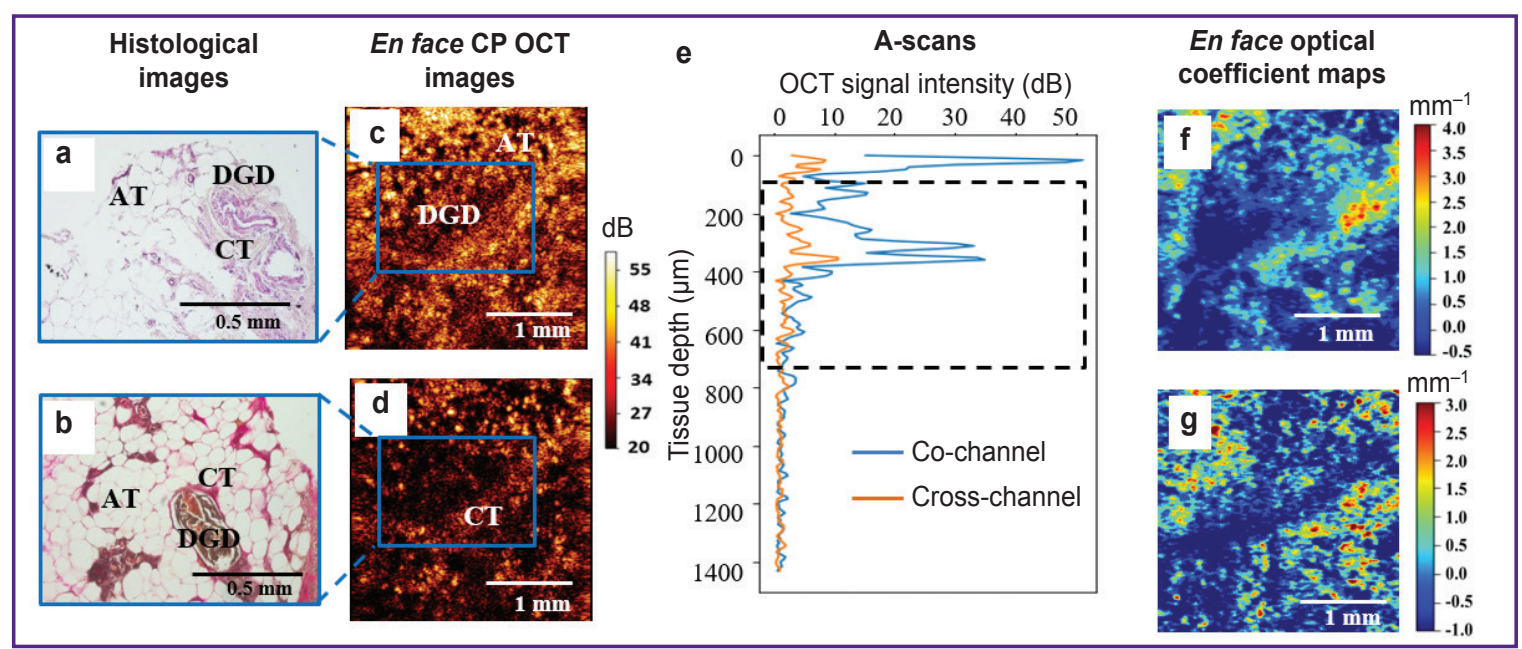

Figure 1. Non-tumor breast tissue

Histological H\&E (a) and van Gieson's (b) stained images; en face structural CP OCT images in co- (c) and cross-polarization (d) channels; random A-scans from 3D data set in co- (blue color) and cross-channels (orange color) (e); corresponding en face color-coded maps of optical coefficients: attenuation coefficient 1 in a crosschannel (f) and difference attenuation coefficient 2 in co- and cross-channels ( $\mathrm{g}$ ). A black dotted rectangular on A-scans indicates the depth range $(80-720 \mu \mathrm{m})$, in which optical coefficients were calculated. CT - connective tissue; AT — adipose tissue; DGD — dilated glandular duct.

The maps of optical coefficients show the following: heterogeneous distribution of coefficient 1 and coefficient 2 values is typical for non-tumor breast tissue. Low values of coefficient 1 (light blue and blue areas) prevailed in the areas of adipose tissue and dilated ducts, while high values (green and yellow-red areas) - in healthy loose connective tissue areas. Coefficient 2 was primarily low and characterized by the distribution of mean values providing connective tissue area imaging (green and yellow-red areas)

prognosis. It is possible to distinguish carcinoma type: solid or sclerosing due to the tissue components, which differently scatter light - connective tissue stroma and agglomerates of tumor cells.

Infiltrative ductal BC of solid structure (with tumor parenchyma dominating over stroma) is characterized by a low heterogeneous OCT signal in co- and crosspolarization channels of CP OCT images (Figure 2 (c), (d)), which is due to densely packed tumor cells in agglomerates that makes tumor tissue poorly lightpermeable. However, there are no anisotropic (fibrous) structures in this cancer subtype (Figure 2 (a), (b)). Such OCT signal distribution enables to easily differentiate visually infiltrative ductal $B C$ of solid structure from nontumor breast tissue.

Infiltrative ductal BC of sclerosing structure. This cancer subtype is characterized by tumor stroma dominating over parenchyma (Figure 3 (a), (b)), OCT signal being distributed heterogeneously (Figure 3 (c), (d)) that makes difficulties for visual differential diagnostics of this cancer subtype with non-tumor breast tissue (though small structures had better contrast in healthy breast images). In corresponding histological specimens, tumor cell clusters are surrounded by tumor stroma fibrous structures with degenerative changes (fibrosis or hyalinosis) resulting in the increase or decrease in OCT signal in a cross-polarization channel. Stroma condition assessment is known to be very important, since tumor collagen matrix plays a key role in $\mathrm{BC}$ invasion and metastasis [32].

\section{Quantitative analysis of scattering and polarization properties of breast tissue and different $B C$ subtypes when making color-coded maps of optical attenuation coefficients in co- and cross-channels}

The approach enabled to sharply enhance of CP OCT image contrast.

Non-tumor breast tissue. Non-tumor breast tissue is characterized by heterogeneous distribution values of coefficient 1 (see Figure 1 (f), (g)). Low values of coefficient 1 (light blue and blue areas) prevailed in the areas of adipose tissue and dilated glandular ducts (less than $0.5 \mathrm{~mm}^{-1}$ ), while high values (over $1.0 \mathrm{~mm}^{-1}$ ) (green and yellow-red areas) prevailed in healthy loose connective tissue areas. It is significant that the images with distribution of coefficient 1 are more contrast than standard CP OCT images, and visualize transparent ducts with a rim created by the duct wall that complied with histological data. Coefficient 2 in this tissue type is, primarily, not high (less than $1.5 \mathrm{~mm}^{-1}$ ) and characterized by mean values providing the imaging of healthy connective tissue areas (green and yellow-red areas). 


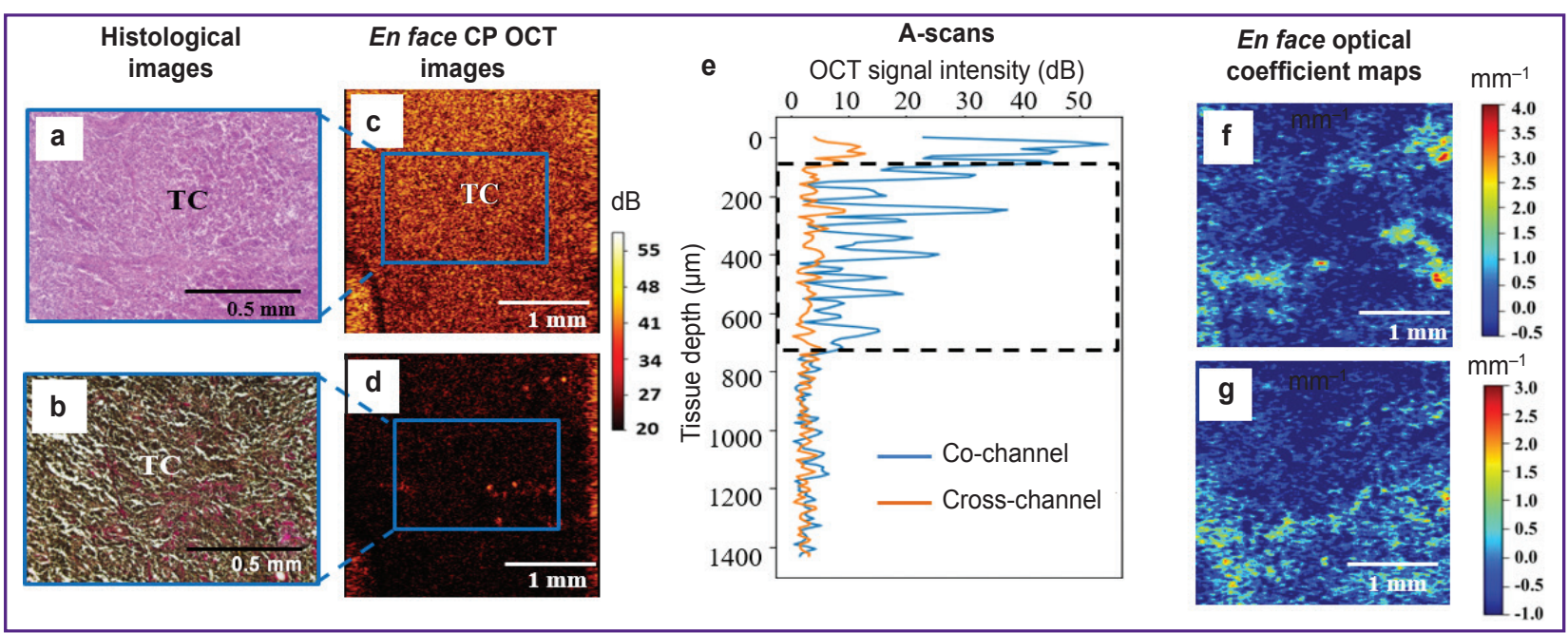

Figure 2. Infiltrative ductal BC of solid structure

Histological H\&E (a) and van Gieson's (b) stained images; en face structural CP OCT images in co- (c) and crosspolarization (d) channels; random A-scans from three-dimensional data set in co- (blue color) and cross-channels (orange color) (e); corresponding en face color-coded maps of optical coefficients: attenuation coefficient 1 in a cross-channel (f) and difference attenuation coefficient 2 in co- and cross-channels $(\mathrm{g})$. A black dotted rectangular on A-scans indicates the depth range $(80-720 \mu \mathrm{m})$, in which optical coefficients were calculated. TC — tumor cells.

This cancer type significantly differs from other studied BC subtypes by its color-coded maps of both coefficients. It is characterized by homogeneous distribution of lowest values of both: coefficient 1 (less than $1.0 \mathrm{~mm}^{-1}$ ) and coefficient 2 (less than $0.5 \mathrm{~mm}^{-1}$ ) (blue and light blue color, no yellow-red color)

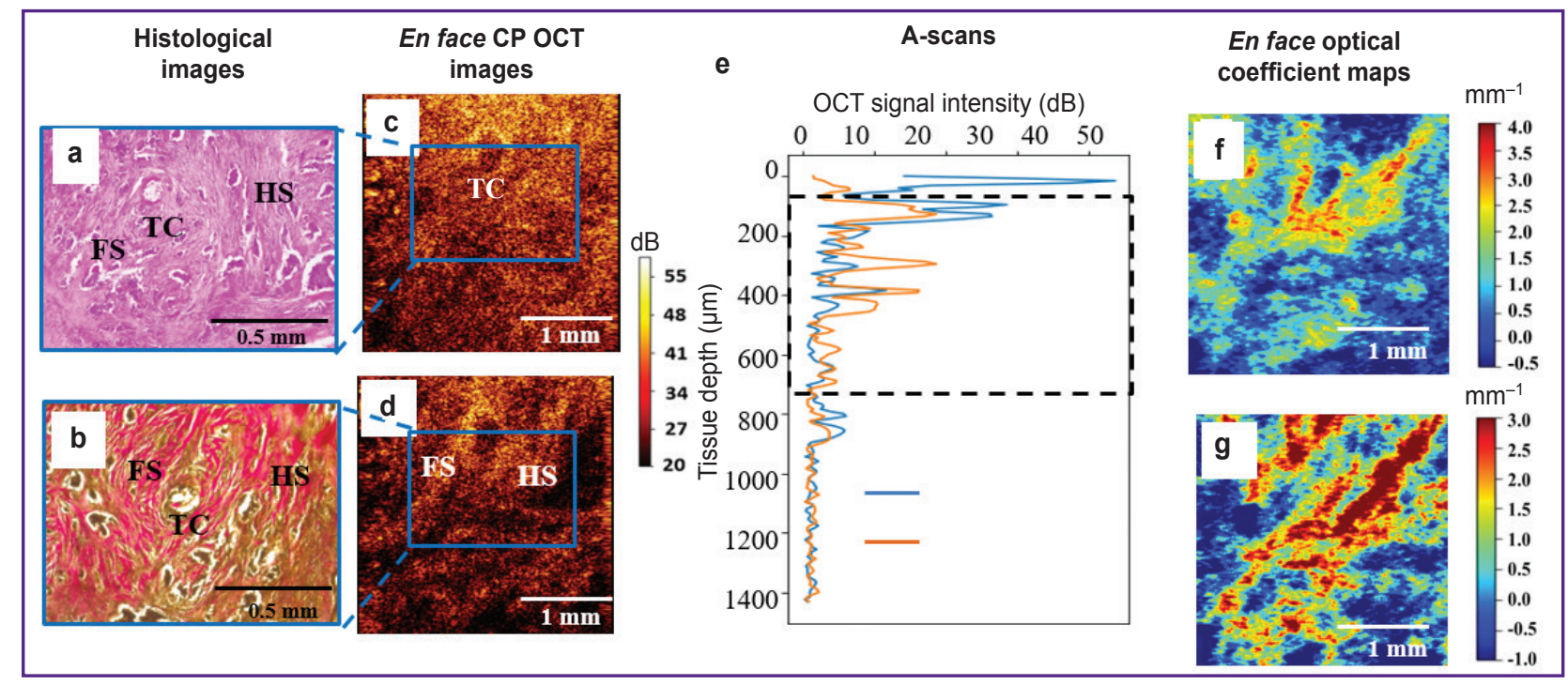

Figure 3. Infiltrative ductal BC of sclerosing structure

Histological H\&E (a) and van Gieson's (b) stained images; en face structural CP OCT images in co- (c) and cross-polarization (d) channels; random A-scans from three-dimensional data set in co- (blue color) and cross-channels (orange color) (e); corresponding en face color-coded maps of optical coefficients: attenuation coefficient 1 in a cross-channel (f) and difference attenuation coefficient 2 in co- and cross-channels $(\mathrm{g})$. A black dotted rectangular on A-scans indicates the depth range (80$720 \mu \mathrm{m}$ ), in which optical coefficients were calculated. TC — tumor cells; FS — fibrous stroma; HS — hyalinized stroma.

In this BC type there is the richest in contrast heterogeneous distribution of high values of both coefficients: coefficient 1 and coefficient 2 (green and yellow-red areas)

Infiltrative ductal BC of solid structure. This cancer subtype differs significantly from other studied BC subtypes by its color-coded maps of both coefficients. It is characterized by homogeneous distribution of lowest values of both: coefficient 1 (less than $1.0 \mathrm{~mm}^{-1}$ ) and coefficient 2 (less than $0.5 \mathrm{~mm}^{-1}$ ) (blue and light blue color, no yellow-red color) (see Figure 2 (f), (g)). 
Infiltrative ductal BC of sclerosing structure.

There is the most contrast heterogeneous distribution of high values of both: coefficient 1 and coefficient 2 (in the range 1.0-3.0 $\mathrm{mm}^{-1}$ ) (see Figure $3(\mathrm{f}),(\mathrm{g})$ ).

However, coefficient 1 and coefficient 2 values in cancer of sclerosing structure depend on tumorous stroma collagen fibers (Figure 4). It is important that fibrous connective tissue in marked hyalinosis becomes dense, and coefficient 1 increases (over $2.5 \mathrm{~mm}^{-1}$ ) (areas of yellow-red color) compared to fibrosis condition, when coefficient 1 is lower (less than $2.0 \mathrm{~mm}^{-1}$ ) (green-blue areas). Coefficient 2, which clearly demonstrates tumor stroma connective tissue state, is as follows: in fibrous changes its high values (in the range 1.0-3.0 $\mathrm{mm}^{-1}$ ) have heterogeneous distribution (yellow-red areas); in hyalinosis of tumor stroma its values, in contrast, decrease (less than $0.5 \mathrm{~mm}^{-1}$ ) (blue, light blue, and green areas). It is related to collagen destruction, the formation of homogeneous semi-opaque dense masses (similar to hyaline) and, therefore, low ability to cross-scattering.

Thus, color-coded maps of BC tissue images based on coefficient 1, compared to structural CP OCT images provide high contrast of healthy and tumor breast tissue (primarily, of solid structure). Coefficient 2 distribution enables to indicate specific features of the stroma state of the sclerosing structure tumors by a high value in the fibrous stroma area. A low value of coefficient 2 indicates to the area of hyalinosis.

Figure 5 shows the resulting distribution histograms of two optical coefficients for study groups. Since non-tumor breast adipose, glandular and connective tissues have different scattering ability, there is heterogeneous distribution of both high and low values of optical coefficients (Figure 5, blue curves). Infiltrative solid BC, which predominantly consists of tumor cells, is characterized by dominating low values of optical coefficients, and therefore, low scattering and polarization properties (Figure 5, orange curves). Heterogeneous distribution of primarily high values of optical coefficients was found in infiltrative ductal BC of sclerosing structure that leads to higher backward
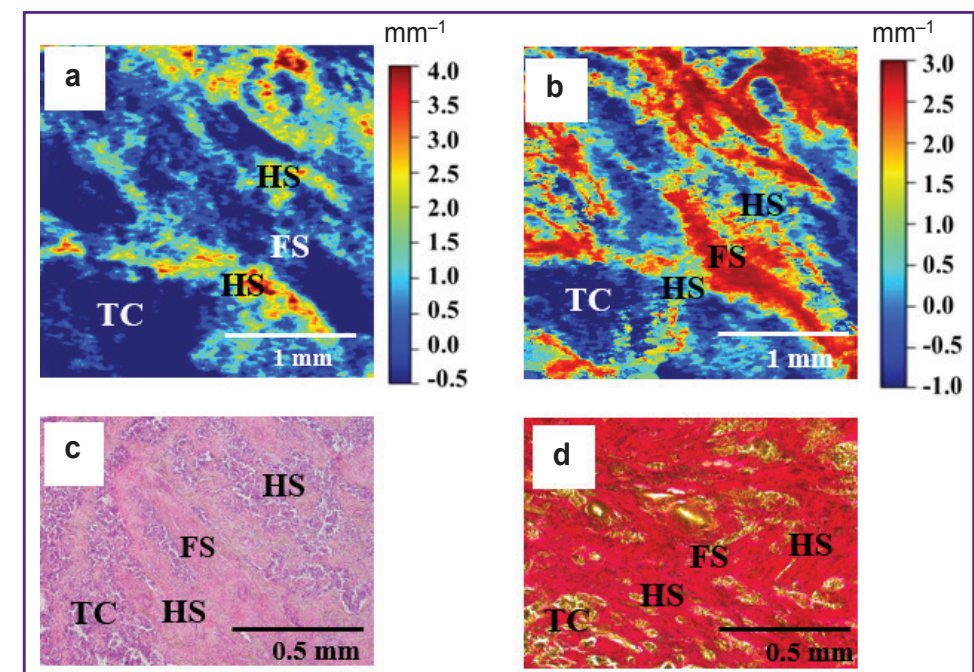

Figure 4. En face color-coded maps of BC tissue with tumor stroma fibrosis and hyalinosis

Optical attenuation coefficient 1 in a cross-channel (a) and difference attenuation coefficient 2 in co- and cross-channels (b). Histological H\&E (c) and van Gieson's (d) stained images. TC — tumor cells; FS fibrous stroma; HS - hyalinized stroma.

In marked hyalinosis, fibrous connective tissue becomes dense and coefficient 1 (a) grows (over $2.5 \mathrm{~mm}^{-1}$ ) (areas of yellow-red color), and in fibrosis its value is lower (less than $2.0 \mathrm{~mm}^{-1}$ ) (green-blue area). Coefficient 2 (b), which indicates tumor stroma connective tissue condition, is as follows: in fibrous changes the distribution of its high values is heterogeneous (in the range $1.0-3.0 \mathrm{~mm}^{-1}$ ) (yellow-red areas); in tumor stroma hyalinosis its values, in contrast, decrease (less than $0.5 \mathrm{~mm}^{-1}$ ) (blue, light blue, and green colors)

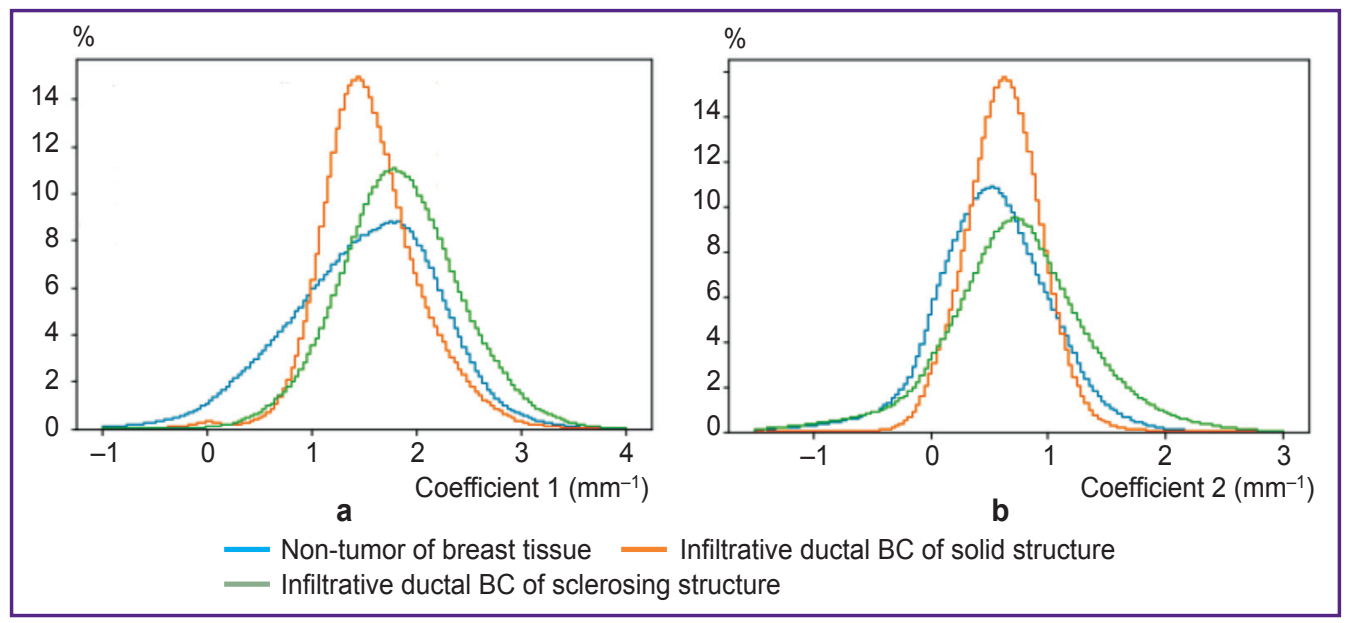

Figure 5. Histograms of the attenuation coefficient 1 (a) and coefficient 2 (b) data for nontumor and breast tumor tissue 
scattering, as well as more expressed polarization effects (Figure 5, green curves).

\section{Conclusion}

CP OCT enables to differentiate the main structural breast tissue components (adipose tissue, connective tissue, and tumor cells) with high contrast by OCT signal in a cross-polarization channel and by the values of optical attenuation coefficients.

The color-coded maps of coefficient 1 and coefficient 2 distributions made by en face CP OCT images of breast tissue samples enable:

easily, to a high accuracy, differentiate non-tumor and tumor breast tissue;

obtain relevant information on the relation and condition of cellular and stromal components in different morphological BC subtypes.

High values of optical coefficients (coefficient 1 and coefficient 2) were found to indicate high content of healthy connective tissue or tumor fibrous stroma. In contrast, their low values suggest tumor cellular component dominating. A difference attenuation coefficient in co- and cross-channels (coefficient 2) enables to indicate and give more evidence on various degenerative changes of tumorous stroma collagen structures. Its low values suggest tumorous stroma hyalinosis growth providing the evidence of secondary deep (degenerative) changes.

A quantitative analysis of scattering and polarization properties of different morphological BC subtypes by calculating optical attenuation coefficients of OCT signal in co- and cross-polarization channels followed by making color-coded maps can serve the basis for independent automated intra-operative diagnosis of different morphological subtypes of infiltrative BC, as well as for the accurate determination of intra-operative resection margin in lumpectomy. Intra-operative identification of positive tumor margins will reduce the number of tumor recurrences and the necessity for reoperations.

Study funding. The study of scattering and polarization properties of breast cancer was supported by the Russian Science Foundation, agreement No.18-75-10068. The calculation technique of optical coefficients was supported by Russian Foundation of Fundamental Research project 16-32-60178 mol_a_dk.

Conflicts of interest. The authors declare no conflicts of interest related to the present study.

\section{References}

1. https://www.cancer.org/research/cancer-facts-statistics/ breast-cancer-facts-figures.html.

2. https://media.nenaprasno.ru/cancer-catalog/additionalarticles/1652/.

3. Zlokachestvennye novoobrazovaniya v Rossii v 2017 godu (zabolevaemost' $i$ smertnost') [Malignant neoplasms in Russia in 2017 (morbidity and mortality)]. Pod red. Kaprina A.D., Starinskogo V.V., Petrovoy G.V. [Kaprin A.D., Starinskiy V.V., Petrova G.V. (editors)]. Moscow: MNIOI im. P.A. Gertsena - filial FGBU "NMITs radiologii" Minzdrava Rossii; 2018; 250 p.

4. Aleskandarany M.A., Vandenberghe M.E., Marchiò C., Ellis I.O., Sapino A., Rakha E.A. Tumour heterogeneity of breast cancer: from morphology to personalised medicine. Pathobiology 2018; 85(1-2): 23-34, https://doi. org/10.1159/000477851.

5. WHO classification of tumours of the breast. Edited by Lakhani S.R., Ellis I.O., Schnitt S.J., Tan P.H., van de Vijver M.J. International Agency for Research on Cancer; 2012.

6. Cèfaro G.A., Genovesi D., Marchese R., Ursini L.A., Cianchetti E., Ballone E., Di Nicola M. Predictors of local recurrence after conservative surgery and whole-breast irradiation. Breast Cancer Res Treat 2006; 98(3): 329-335, https://doi.org/10.1007/s10549-006-9169-0.

7. Luini A., Rososchansky J., Gatti G., Zurrida S., Caldarella P., Viale G., Rosali dos Santos G., Frasson A. The surgical margin status after breast-conserving surgery: discussion of an open issue. Breast Cancer Res Treat 2008; 113(2): 397-402, https://doi.org/10.1007/s10549-008-9929-0.

8. Catalano O.A., Horn G.L., Signore A., lannace C., Lepore M., Vangel M., Luongo A., Catalano M., Lehman C., Salvatore M., Soricelli A., Catana C., Mahmood U., Rosen B.R. PET/MR in invasive ductal breast cancer: correlation between imaging markers and histological phenotype. Br J Cancer 2017; 116(7): 893-902, https://doi. org/10.1038/bjc.2017.26.

9. Kuhl C.K. Abbreviated magnetic resonance imaging (MRI) for breast cancer screening: rationale, concept, and transfer to clinical practice. Annu Rev Med 2019; 70(1): 501519, https://doi.org/10.1146/annurev-med-121417-100403.

10. Taylor K.J., Merritt C., Piccoli C., Schmidt R., Rouse G., Fornage B., Rubin E., Georgian-Smith D., Winsberg F., Goldberg B., Mendelson E. Ultrasound as a complement to mammography and breast examination to characterize breast masses. Ultrasound Med Biol 2002; 28(1): 19-26, https://doi. org/10.1016/s0301-5629(01)00491-4.

11. Evans A., Trimboli R.M., Athanasiou A., Balleyguier C. Baltzer P.A., Bick U., Camps Herrero J., Clauser P., Colin C., Cornford E., Fallenberg E.M., Fuchsjaeger M.H., Gilbert F.J., Helbich T.H., Kinkel K., Heywang-Köbrunner S.H., Kuhl C.K., Mann R.M., Martincich L., Panizza P., Pediconi F., Pijnappel R.M., Pinker K., Zackrisson S., Forrai G., Sardanelli F.; European Society of Breast Imaging (EUSOBI), with language review by Europa Donna-The European Breast Cancer Coalition. Breast ultrasound: recommendations for information to women and referring physicians by the European Society of Breast Imaging. Insights Imaging 2018; 9(4): 449-461, https://doi.org/10.1007/s13244-018-0636-z.

12. Esbona K., Li Z., Wilke L.G. Intraoperative imprint cytology and frozen section pathology for margin assessment in breast conservation surgery: a systematic review. Ann Surg Oncol 2012; 19(10): 3236-3245, https://doi.org/10.1245/ s10434-012-2492-2.

13. Yao X., Gan Y., Chang E., Hibshoosh H., Feldman S., Hendon C. Visualization and tissue classification of human breast cancer images using ultrahigh-resolution OCT. Lasers Surg Med 2017; 49(3): 258-269, https://doi.org/10.1002/ Ism.22654.

14. Zhou C., Cohen D.W., Wang Y., Lee H.C., 
Mondelblatt A.E., Tsai T.H., Aguirre A.D., Fujimoto J.G., Connolly J.L. Integrated optical coherence tomography and microscopy for ex vivo multiscale evaluation of human breast tissues. Cancer Res 2010; 70(24): 10071-10079, https://doi. org/10.1158/0008-5472.can-10-2968.

15. Ha R., Friedlander L.C., Hibshoosh H., Hendon C., Feldman S., Ahn S., Schmidt H., Akens M.K., Fitzmaurice M., Wilson B.C., Mango V.L. Optical coherence tomography: a novel imaging method for post-lumpectomy breast margin assessment-a multi-reader study. Acad Radiol 2018; 25(3): 279-287, https://doi.org/10.1016/j.acra.2017.09.018.

16. Nguyen F.T., Zysk A.M., Chaney E.J., Kotynek J.G., Oliphant U.J., Bellafiore F.J., Rowland K.M., Johnson P.A., Boppart S.A. Intraoperative evaluation of breast tumor margins with optical coherence tomography. Cancer Research 2009; 69(22): 8790-8796, https://doi.org/10.1158/0008-5472.can-084340.

17. Savastru D., Chang E.W., Miclos S., Pitman M.B., Patel A., Iftimia N. Detection of breast surgical margins with optical coherence tomography imaging: a concept evaluation study. J Biomed Opt 2014; 19(5): 056001, https://doi. org/10.1117/1.jbo.19.5.056001.

18. Mujat M., Ferguson R.D., Hammer D.X., Gittins C., Iftimia N. Automated algorithm for breast tissue differentiation in optical coherence tomography. J Biomed Opt 2009; 14(3): 034040, https://doi.org/10.1117/1.3156821.

19. Zysk A.M., Boppart S.A. Computational methods for analysis of human breast tumor tissue in optical coherence tomography images. J Biomed Opt 2006; 11(5): 054015, https://doi.org/10.1117/1.2358964.

20. Zysk A.M., Chaney E.J., Boppart S.A. Refractive index of carcinogen-induced rat mammary tumours. Phys Med Biol 2006; 51(9): 2165-2177, https://doi.org/10.1088/0031-9155/51/9/003.

21. Hsiung P.-L., Phatak D.R., Chen Y., Aguirre A.D., Fujimoto J.G., Connolly J.L. Benign and malignant lesions in the human breast depicted with ultrahigh resolution and three-dimensional optical coherence tomography. Radiology 2007; 244(3): 865-874, https://doi.org/10.1148/ radiol.2443061536.

22. South F.A., Chaney E.J., Marjanovic M., Adie S.G., Boppart S.A. Differentiation of ex vivo human breast tissue using polarization-sensitive optical coherence tomography. Biomed Opt Express 2014; 5(10): 3417, https://doi. org/10.1364/boe.5.003417.

23. Patel R., Khan A., Quinlan R., Yaroslavsky A.N. Polarization-sensitive multimodal imaging for detecting breast cancer. Cancer Res 2014; 74(17): 4685-4693, https://doi. org/10.1158/0008-5472.can-13-2411.

24. Chin L., Latham B., Saunders C.M., Sampson D.D., Kennedy B.F. Simplifying the assessment of human breast cancer by mapping a micro-scale heterogeneity index in optical coherence elastography. J Biophotonics 2016; 10(5): 690-700, https://doi.org/10.1002/jbio.201600092.

25. Kennedy B.F., McLaughlin R.A., Kennedy K.M., Chin L., Wijesinghe P., Curatolo A., Tien A., Ronald M., Latham B., Saunders C.M., Sampson D.D. Investigation of optical coherence microelastography as a method to visualize cancers in human breast tissue. Cancer Res 2015; 75(16): 3236-3245, https://doi.org/10.1158/0008-5472.

26. Gubarkova E.V., Sovetsky A.A., Zaitsev V.Y., Matveyev A.L., Vorontsov D.A., Sirotkina M.A., Matveev L.A., Plekhanov A.A., Pavlova N.P., Kuznetsov S.S., Vorontsov A.Y., Zagaynova E.V., Gladkova N.D. OCT-elastography-based optical biopsy for breast cancer delineation and express assessment of morphological/molecular subtypes. Biomed Opt Express 2019; 10(5): 2244, https://doi.org/10.1364/ boe.10.002244.

27. Gelikonov V.M., Romashov V.N., Shabanov D.V., Ksenofontov S.Y., Terpelov D.A., Shilyagin P.A., Gelikonov G.V., Vitkin I.A. Cross-polarization optical coherence tomography with active maintenance of the circular polarization of a sounding wave in a common path system. Radiophys Quantum El 2018; 60(11): 897-911, https://doi.org/10.1007/s11141-018-9856-9.

28. Gubarkova E.V., Dudenkova V.V., Feldchtein F.I., Timofeeva L.B., Kiseleva E.B., Kuznetsov S.S., Shakhov B.E., Moiseev A.A., Gelikonov V.M., Gelikonov G.V., Vitkin A., Gladkova N.D. Multi-modal optical imaging characterization of atherosclerotic plaques. J Biophotonics 2015; 9(10): 10091020, https://doi.org/10.1002/jbio.201500223.

29. Kiseleva E., Kirillin M., Feldchtein F., Vitkin A., Sergeeva E., Zagaynova E., Streltzova O., Shakhov B., Gubarkova E., Gladkova N. Differential diagnosis of human bladder mucosa pathologies in vivo with cross-polarization optical coherence tomography. Biomed Opt Express 2015; 6(4): 1464-1476, https://doi.org/10.1364/boe.6.001464.

30. Rak molochnoy zhelezy. Morfologicheskaya diagnostika i genetika [Breast cancer. Morphological diagnosis and genetics]. Pod red. Franka G.A., Zavalishinskoy L.E., Pozharisskogo K.M. [Frank G.A., Zavalishinskaya L.E., Pozharisskiy K.M. (editors)]. Moscow: Prakticheskaya meditsina; 2014.

31. Kut C., Chaichana K.L., Xi J., Raza S.M., Ye X., McVeigh E.R., Rodriguez F.J., Quiñones-Hinojosa A., Li X. Detection of human brain cancer infiltration ex vivo and in vivo using quantitative optical coherence tomography. Sci Trans/ Med 2015; 7(292): 3010611, https://doi.org/10.1126/ scitranslmed.3010611.

32. Provenzano P.P., Eliceiri K.W., Campbell J.M., Inman D.R., White J.G., Keely P.J. Collagen reorganization at the tumor-stromal interface facilitates local invasion. BMC Med 2006; 4(1): 38, https://doi.org/10.1186/1741-7015-4-38. 